\title{
Thermodynamic Equilibrium for Mixtures of Combustible Gases and Air
}

\author{
Richard Martin Gibbons \\ Received: July 07, 2016 / Accepted: July 15, 2016 / Published: September 30, 2016.
}

\begin{abstract}
Standard treatments of thermodynamic equilibrium are incomplete. They do not take account of all factors determining equilibrium, cannot explain why many systems do not reach equilibrium and do not discuss the questions of reaching and maintaining equilibrium. The arguments presented here provide a single physical definition of thermodynamic equilibrium that accounts for all factors determining thermodynamic equilibrium for mixtures of combustible gases and air. Based on the standard definition of thermodynamic equilibrium, the MBD (Maxwell Boltzmann distribution) and a simple molecular model lead to three possible types of equilibrium. The regions of temperature pressure and composition for each type of equilibrium are defined by the measured values of ignition temperatures and the explosive and flammability limits. How this definition of thermodynamic equilibrium can be extended to all molecular systems is discussed in the following papers.
\end{abstract}

Key words: Thermodynamics, equilibrium, Gibbs function, statistical mechanics, thermo-physical properties.

\section{Introduction}

The definition of thermodynamic equilibrium is a basic concept in chemistry for all theories and measurements. So it is surprising that there is no single definition of it which is applicable to all equilibriums [1-3].

The purpose of this article is to show that there is a single definition of thermodynamic equilibrium for the class of mixtures of combustible gases and air. There are extensive sets of experimental data for these systems [4-6] which specify ranges of temperature, pressure and composition for which different types of equilibrium occur that are all described by the same molecular definition of equilibrium but with different controlling factors.

The two standard definitions of thermodynamic equilibrium are based either on the global minimum of the Gibbs function at the conditions of the molecular system, or kinetic controlled equilibrium at a local minimum in the Gibbs function at that state. These

Corresponding author: Richard Martin Gibbons, Ph.D., DIC retired, research fields: thermodynamics, statistical mechanics and thermo-physical properties. definitions are based solely on equilibrium properties and hence cannot account for either all the factors governing equilibrium or whether it can be reached and maintained. The analysis below also shows that kinetic controlled equilibrium is defined incompletely.

The molecular and statistical basis of the new definition is set out below and is followed by a discussion of its application to experimental data for mixtures of combustible gases and air. These data provide experimental confirmation that the global minimum in the Gibbs function applies only to those equilibriums where non thermodynamic factors do not control equilibrium. Where non thermodynamic factors control equilibrium, which is probably the majority of cases, physical factors give rise to the three possible options which are introduced in the new definition of thermodynamic equilibrium discussed below.

\section{The Definition of Thermodynamic Equilibrium for Combustible Gases}

Standard definitions of thermodynamic equilibrium are based on equilibrium properties of the molecular systems. 
The first definition is: A molecular system at equilibrium has constant uniform values of temperature, pressure and composition and the Gibbs function is a minimum.

When there are means for producing and maintaining constant conditions, and there are no impediments which could prevent molecules from reaching their equilibrium energies, this statement describes thermodynamic equilibrium correctly.

When there are impediments preventing some of the molecules from reaching their equilibrium energies at the condition where the molecular system has the global minimum value of the Gibbs function, the definition for kinetic controlled equilibrium is used instead. The Gibbs function then is a minimum for the system over the molecular energies accessible to it at the conditions of the system but the standard definition does not discuss what these impediments could be.

The deficiencies of these two statements lie in that they do not contain any basis for including non thermodynamic factors that control whether a system can or cannot reach equilibrium. Nor do they address the question of whether the equilibrium can be reached and be maintained. Those who say thermodynamics does not necessarily involve molecules have no way of accounting for why molecular systems do or do not reach equilibrium, for these all involve non thermodynamic factors arising from molecular interactions. These two definitions are therefore incomplete.

The definition proposed here is based on the MBD (Maxwell Boltzmann distribution) and a simple statistical model which is supported by the data for mixtures of combustible gases and air.

A molecular system is at equilibrium when the molecules have an MBD for the accessible energies of the molecules, the Gibbs function is a minimum, the system has constant values of temperature, pressure and composition and there are means of reaching and maintaining that equilibrium.

All reactions occur as a result of the collisions of molecules with sufficient energy to effect an exchange or transfer of electrons. The simple statistical model is based on the accessible energy levels of the molecules. These are determined by the average kinetic energy of the molecules, which arises from the MBD, at the temperature of the system. Three possible options can occur for combustible gas mixtures:

Option 1: There are enough molecules with sufficient kinetic energy for a reaction to proceed;

Option 2: All the molecules have enough kinetic energy for the reaction to proceed and an explosion occurs;

Option 3: There are insufficient molecules with enough kinetic energy for the reaction to proceed and it ceases or does not start.

Examples of these three types of behaviour are demonstrated using data for mixtures of combustible gases and air.

But before applying these ideas to experimental data some discussion of "accessible molecular energy levels available to molecules at a given temperature" is needed. For an energy level to be accessible to a system it must contain enough molecules with high enough kinetic energies to be able to contribute to the properties and any changes the system undergoes. The simplest way of estimating the number of molecules with high enough energy is to use the normal distribution tables and the central limit theorem [7].

The MBD is not a normal distribution, but like nearly all distributions, can be transposed into an approximately normal distribution by expressing it in terms of $\left(u_{i}-u_{a v}\right) / \sigma$, where $u_{a v}$ is the average energy, $u_{i}$ is the energy of $i$ th molecule and $\sigma$ is the standard deviation. The value of the kinetic energy $u_{a v}$ is equal to $1.5 \mathrm{kT}$ for an ideal gas. Less well known is that $\sigma$ for the MBD of an ideal gas has the same value. It follows from the central limit theorem that the probability of a molecule having a particular energy can be obtained from the normal probability table. Hence $97.5 \%$ of the molecules have energies of $<2 u_{a v}$ and $99.99 \%<5 u_{a v}$. 
The term "enough molecules for a reaction to continue" also requires some explanation. There is no rigorous method of calculating how many molecules are enough. It must be established experimentally though the data for mixtures of combustible gases and air provide some empirical estimates as to their likely values. This is discussed further below.

\section{The Equilibrium Behaviour of Mixtures of Combustible Gases and Air}

There are extensive tabulations of the behaviour of mixtures of combustible gases and air in chemical and chemical engineering handbooks [4-6] that are essential for the safe handling of these mixtures. They have not previously been used as supporting data for a molecular model of these reactions.

Analysis starts with the ignition temperatures of these mixtures, defined as the temperature at which a combustible gas spontaneously reacts with air. At these temperatures there are no impediments that prevent the molecules in the mixture from reacting freely to produce thermodynamic equilibrium where the Gibbs function is a minimum and the resulting mixture has an MBD of energies, as in option 1. For most combustible gases they are in the region $450-700{ }^{\circ} \mathrm{C}$. Equilibrium for these systems is controlled solely by thermodynamic equilibrium properties.

Thermodynamic equilibrium in all the other cases is determined by non thermodynamic factors.

The controlled burning of a combustible gas corresponds to the first option 1 above. There are enough molecules of combustible gas and air able to react and the reaction proceeds when the mixture is ignited.

However, prior to ignition, the mixture of combustible gas and air is an unreacted mixture below the ignition temperature, in a state where not enough molecules can react for the reaction to proceed, corresponding to option 3 above. Whether there are enough molecules able to react depends on the surroundings, as the following example shows [2].
An equimolar mixture of hydrogen and oxygen at $25^{\circ} \mathrm{C}$ and $1 \mathrm{~atm}$ stays unreacted in a kinetic controlled equilibrium with the molecules in an MBD. Introduce a platinum catalyst and molecules react to form a mixture of hydrogen and oxygen and water vapour. The original MBD is unaffected by the catalyst but the energy levels accessible for the reaction have changed, enabling enough molecules to participate in the reaction for it to proceed at the temperature of the system. This example of kinetic controlled equilibrium shows that a full definition of such equilibriums must include the surroundings. There is further discussion of this below.

The next property to be discussed is the flammability limit. This is the maximum concentration of a combustible gas in air which will not react at a given temperature as in option 3. The flammability limit defines the concentrations of molecules that are enough for the reaction to proceed for these systems at the temperature of the system. Generally these values are in the range $1-5 \%$.

These values are critical for the safe distribution of natural gas to the public. Gas is odorised to be detectable at a concentration of $10 \%$ of the flammability limit. Many of these systems also have upper flammability limits where there is insufficient oxygen to support combustion. These values define the condition of option 3 above where there are not enough oxygen molecules to support combustion.

The most important regions are those defined by the explosion limits which define the regions of temperature pressure and composition where the mixtures explode if ignited. Explosion limits define the conditions for option 2 above when all the molecules react virtually simultaneously which is as fast as the system can react.

This discussion shows that the three options listed provide molecular interpretations of the real physical conditions defined by the data for these systems. They have implications for the standard definition of kinetic controlled equilibrium which is discussed next. 


\section{Kinetic Controlled Equilibrium}

Kinetic controlled equilibrium is the second definition of equilibrium given above. Callen [3] and others have noted this definition leads to a circular argument as to what defines thermodynamic equilibrium. Any system with a minimum Gibbs function and constant uniform values of temperature, pressure and composition is at thermodynamic equilibrium. The circularity arises because the equilibrium is defined solely in terms of equilibrium properties of the system and takes no account of the non-thermodynamic properties of the molecules that actually control these equilibriums. The system of hydrogen and oxygen discussed above makes this clear. There is an unstated condition that the hydrogen and oxygen cannot react at the conditions of the system, whether it can depend on the surroundings or not. The definition of kinetic controlled equilibrium uses the temperature and pressure as a proxy for the activation energy barriers that prevent the reaction from occurring. It is this which produces the circular argument.

This circularity can be avoided in two ways. Simply say that, the equilibrium is dependent on no reaction occurring at the state of the system. Alternatively define the energy barrier that has to be overcome for a reaction to proceed. For the reactions to proceed at these conditions require concentrations of molecules with energies larger than the activation energy which normally prevent transfers of electrons in molecular collisions.

The sizes of the activation energies depend on the molecular properties of the molecules. The number of molecules able to successfully overcome these barriers depends on the system conditions and the MBD.

The suggestion that equilibrium is defined as no reaction occurs at the system temperature avoids any circular argument but does not say why no reaction occurs. The alternative also avoids any circular arguments but leaves the activation energy value undefined. Both options show that the definition of kinetic controlled equilibrium is incomplete and offers no guidance as to how the system will respond with changes of conditions.

\section{Discussion}

The process of ignition, the data for the ignition temperatures and the flammability limits show, why there must be a minimum number of molecules involved for a reaction to proceed and suggests what ranges of concentrations will be required.

To ignite these mixtures energy is provided, via an electric spark or flame, to a small volume of the gas, raising its temperature to the ignition temperature and ignites it. The energy from those reactions, heats adjacent volumes to the ignition temperature and the reaction propagates. Successful ignition of the flammable gas and air mixture heats both reacting molecules and the inert gases present (mainly nitrogen). If insufficient heat is released, the ignition temperature is not reached in the adjacent volumes and the reaction ceases.

The values of the flammability limits suggest that the minimums for "concentrations of molecules for a reaction to proceed" will be of the order a few percent. In turn, this suggests that the range of energies in the MBD that will take part in the reaction are those where, $u_{i} / u_{a v}<4$.

\section{Conclusions}

The definition introduced above gives a single physical description of all equilibriums for these systems. They are all defined by minimums in the Gibbs function, constant conditions of the temperature pressure and composition and the MBD.

The definition of thermodynamic equilibrium in terms of the global minimum of the Gibbs function applies only to those equilibriums where no other factors prevent equilibrium from being reached. These equilibriums are determined solely by mathematics. Kinetic controlled equilibrium is determined by a combination of mathematical and chemical factors. The definition of kinetic controlled equilibrium should 
be modified to include all the factors that determine equilibrium.

The three options above account for all types of equilibrium for these mixtures of flammable gases and air. Is there a similar definition of thermodynamic equilibrium for all types of systems? There is but it requires a redefinition of one of the processes involved which is discussed in the following papers.

\section{References}

[1] Smith, E. B. 1993. Basic Chemical Thermodynamics.
Oxford: Oxford University Press.

[2] Pitzer, K. E., and Brewer, L. 1961. Thermodynamics. New York: McGraw Hill.

[3] Callen, H. B. 1961. Thermodynamics. New York: McGraw Hill.

[4] Perry, J. H., and Chilton, C. H. 1973. Chemical Engineers Handbook, 5th edition. New York: McGraw Hill.

[5] Haynes, W. M. 2012. CRC Handbook of Chemistry and Physics, 93rd edition. London: Taylor and Francis.

[6] Yaws, C. L. 2001. Matheson Gas Data Book, 7th edition. New York: McGraw Hill.

[7] Attwood, G., and Dyer, G. 1995. Statistics 2. Heinemann, 26-74. 